\title{
Labeling of Acetaminophen with I-131 and Biodistribution in Rats
}

\author{
Fatma Yurt Lambrecht, ${ }^{*, a}$ Kubra Durkan, ${ }^{b}$ Yeliz Yildirim, ${ }^{c}$ and Cigdem $\operatorname{AcAR}^{a}$ \\ ${ }^{a}$ Department of Nuclear Applications, Institute of Nuclear Sciences, Ege University; Bornova, 35100, Izmir, Turkey: \\ ${ }^{b}$ Chemistry Program, Izmir Vocational School, Dokuz Eylul University; Buca, 35160, Izmir, Turkey: and ${ }^{c}$ Department of \\ Chemistry, Faculty of Science, Ege University; Bornova, 35100, Izmir, Turkey.
}

Received August 12, 2005; accepted October 24, 2005

The aim of the present study was to label acetaminophen (APAP) with I-131 and to determine its radiopharmaceutical potential in rats. Acetaminophen was labeled with I-131 using the iodogen method. The radiochemical purity of ${ }^{131}$ I-APAP was determined by RTLC and paper electrophoresis. The labeling yield was $94 \pm 4 \%$. The biodistribution studies of the labeled compound (specific activity; $56.60 \mathrm{GBq} / \mathrm{mmol}$ ) were performed in male Albino Wistar rats. The uptake of ${ }^{131}$ I-APAP in some organs were determined at different time after injection to the rats. The radioactivity in each organ was counted and the percentage of injected activity per gram of tissue weight (\%ID/g) for each organ and blood was calculated. ${ }^{131}$ I-APAP uptake in the lung, liver, kidneys, pancreas, blood, stomach and some brain region, were observed. Thus, ${ }^{131}$ I-APAP may be radiopharmaceutical for the imaging of the brain.

Key words acetaminophen; I-131; radiolabel; ${ }^{131}$ I-acetaminophen $\left({ }^{131} \mathrm{I}\right.$-ADAP)

Paracetamol has been named as 4-hydroxyacetanilide or $N$-acetyl- $p$-aminophenol and in the U.S. pharmacopoeia, it is known as acetaminophen (APAP). APAP has good analgesic and antipyretic properties. ${ }^{1-3)}$ It is widely used as an oral nonprescription drug and is frequently used with other analgesic and antipyretic drugs as a multiple-drug therapy. ${ }^{4)}$ Also, it is suitable for the treatment of pains of all kinds (headaches, dental pain, postoperative pain, pain in connection with colds, post-traumatic muscle pain). APAP is well suited for use in children. It represents a preferred alternative when aspirin is contraindicated (e.g. because of a history of ulcer or viral infection in the child). Migraine headaches, dysmenorrheal and joint pain can also be influenced advantageously. APAP has been compared to many other analgesics and is considered approximately equipotent to aspirin (acetylsalicylic acid). In general, APAP is less efficacious than salicylates and other antirheumatic agents for problems that require anti-inflammatory treatment. ${ }^{5)}$ APAP relieves pain by elevating the pain threshold, that is, by requiring a greater amount of pain to develop before it is felt by a person. It reduces fever through its action on the heat regulating center of the brain. ${ }^{6}$ Several studies which have shown that APAP crosses blood-brain barrier support the views that this drug exerts its effect by a central mechanism. However, they were based on the measurement of acetaminophen levels either in cerebrospinal fluid (CSF), or in total brain tissues following infra-analgesic or toxic doses of the compound. ${ }^{7,8)}$

The aim of the present study was to label APAP with ${ }^{131} \mathrm{I}$ and investigate the radiopharmaceutical potential of ${ }^{131} \mathrm{I}$ APAP in rats.

\section{Experimental}

$\mathrm{Na}^{131} \mathrm{I}$ was supplied from the Department of Nuclear Medicine of Ege University. Iodogene and APAP were purchased from Sigma Co. All other chemicals were purchased from Merck and they were reagent grade.

Radio Thin Layer Chromatography (RTLC) Radio thin layer chromatography was carried out by using cellulose-coated plastic sheets (Merck 5565 ). The plastic sheets with a thickness of $0.1 \mathrm{~mm}$ were cut into $1 \times 10 \mathrm{~cm}$ sheets and they were used for quality control of radiopharmaceutical. The sample was applied at a marked point. Two solvent systems were used for developing (solvent 1: $n$-butanol-water-acetic acid (60/25/15), solvent 2 : $0.9 \% \mathrm{NaCI}$ ). After developing, RTLC sheets were dried and cut into $0.5 \mathrm{~cm}$ strips. The radioactivity of each strip was counted using a $\mathrm{Cd}(\mathrm{Te})$ detector equipped with a RAD 501 single-channel analyzer. RTLC chromatograms were obtained from these counts. Finally, $R f$ values and labeling yields were found.

Conditions of Electrophoresis Paper electrophoresis was performed using a Gelman electrophoresis chamber. Cathode and anode poles and application points were indicated on Whatman papers $(1 \times 25 \mathrm{~cm})$ and the papers were moistened by buffer solution (Borate buffer: $9 \mathrm{~g} / 1$ in water, $\mathrm{pH}$ : 9.1). After the sample was applied to the paper, they were put into the electrophoresis chamber. The standing time and applied voltage were $1 \mathrm{~h}$ and $250 \mathrm{~V}$, respectively. The developed papers were dried and cut into $1 \mathrm{~cm}$ strips. They were counted by a $\mathrm{Cd}(\mathrm{Te})$ detector equipped with a RAD 501 single-channel analyzer. Electrophoresis diagrams were obtained from these counts.

Preparing the Iodogen Coated Tubes A milligram amount of iodogen was dissolved in $\mathrm{CH}_{2} \mathrm{CI}_{2}$ and transferred to closed tubes. $\mathrm{CH}_{2} \mathrm{CI}_{2}$ was evaporated by air flow and iodogen was deposited on the walls of glass tubes as a thin film. These tubes were stored at $0^{\circ} \mathrm{C}$ until use.

Labeling Procedure One milligram APAP was dissolved in 5\% ethanol solution and this solution was placed in an iodogen coated vial. Then, $222 \mathrm{MBq}(6 \mathrm{mCi}) \mathrm{Na}^{131} \mathrm{I}$ was added to the vial. The vial was left at room temperature for $20 \mathrm{~min}$. The reaction was ended by adding $0.1 \mathrm{~N} \mathrm{Na} \mathrm{SO}_{3}$ solution $(100 \mu \mathrm{l})$. The radiochemical yield was determined with RTLC and paper electrophoresis methods. The labeling yield of ${ }^{131}$ I-APAP was determined to be $94 \pm 4.0 \%(n=6)$.

Determination of the Octanol-Water Partition Coefficient A $100 \mu \mathrm{l}$ aliquot of ${ }^{131} \mathrm{I}$-APAP was mixed with $3 \mathrm{ml}$ each of $n$-octanol and $0.1 \mathrm{M}$ phosphate $(\mathrm{pH}=7.4)$ in a test tube. The tube was shaken, incubated for $1 \mathrm{~h}$ at room temperature and centrifuged for $5 \mathrm{~min}$. Then, $0.5 \mathrm{ml}$ aliquots of each phase were removed and counted by a $\mathrm{Cd}(\mathrm{Te})$ detector equipped with a $\mathrm{RAD}$ 501 single-channel analyzer. ${ }^{9)}$

Biodistribution Studies in Rats The Institution Animal Review Committee of Ege University approved the animal experiments. The biodistribution study was performed in male Albino Wistar rats $(100-150 \mathrm{~g})$. Four rats were used for each time point. The $\mathrm{pH}$ of ${ }^{131} \mathrm{I}$-APAP $(0.6 \mathrm{mg} / \mathrm{rat}$ APAP $)$ was adjusted to 7 using $0.1 \mathrm{~N}$ ammonium hydroxide. The solution was passed through a Milipore filter $(0.22 \mu \mathrm{m}$ pore $)$. Then it was injected into the tail vein of male Albino Wistar rats. The rats were sacrificed by ether anaesthesia at $15,30,60$, and $120 \mathrm{~min}$ after injection. The organs and blood were removed and weighed. The brains were excised quickly washed into cold saline solution and dissected into their components. Their activities were counted with a $\mathrm{Cd}(\mathrm{Te})$ detector equipped with a RAD 501 single-channel analyzer. The $\% \mathrm{ID} / \mathrm{g}$ values for each organ and blood were calculated.

Statistical Analyses Statistical analyses of the biodistribution results were performed using commercial software (SPSS 10.0 for Windows). After calculating the activity per gram for each organ, the relationship between the ${ }^{131}$ I-APAP complex and organs was determined statistically by univariate analysis of variance. Statistical significance was taken as $(p<0.05)$. 


\section{Results and Discussion}

Table 1 shows $R f$ values from which RTLC chromatograms were obtained. The experiment results indicated that the labeling yield of ${ }^{131}$ I-APAP was $94 \pm 4.0 \%$ under optimal conditions ( $\mathrm{pH}$ : 6, reaction time: $20 \mathrm{~min}$, reaction temperature: room temperature).

The stability experiment results indicated that the labeling yield of ${ }^{131} \mathrm{I}$-APAP decreased to $93 \pm 1.0 \%$ and $74.65 \pm 1.0 \%$ at the $3 \mathrm{rd}$ and 24 th hours, respectively at room temperature. Hence, the period of stability of ${ }^{131}$ I-APAP is sufficient for imaging procedures.

The result of paper electrophoresis showed that the labeled product has a positive charge. The $n$-octanol/water partition coefficient (lipophilicity) of ${ }^{131} \mathrm{I}$-APAP was determined and the lipophilicity was found to be $7.0 \pm 3.2(n=3)$. As known, the uptake of radiopharmaceutical in the brain depends on a high $n$-octanol/water ratio. ${ }^{9)}$ Figure 1 showed the biodistribution of ${ }^{131} \mathrm{I}$-APAP as $\% \mathrm{ID} / \mathrm{g}$ (mean \pm S.D.) organs for each time point after-injection.

The $\% \mathrm{ID} / \mathrm{g}$ value of the labeled compound in the lung, kidneys, pancreas and blood was 1.15, 1.02, 1.07, and 2.29 at 15 min, respectively (Fig. 1).

Discussion ${ }^{131}$ I-APAP was obtained in a high yield. The data of the biodistribution experiments showed that the highest accumulation was observed in the lung $(p<0.05)$, pancreas $(p<0.05)$, blood $(p<0.05)$ and kidneys, at $15 \mathrm{~min}$. Mugford and Tarloff observed that the uptake of covalent

Table 1. Rf Values

\begin{tabular}{ccc}
\hline \hline & Solvent 1 & Solvent 2 \\
\hline${ }^{131} \mathrm{I}$-APAP & 1.00 & 0.45 \\
$\mathrm{Na}^{131} \mathrm{I}$ & 0.40 & 0.07 \\
\hline
\end{tabular}

Solvent 1: $n$-butanol-water-acetic acid (60/25/15). Solvent 2: $0.9 \% \mathrm{NaCI}$. binding of radiolabeled [ring ${ }^{14} \mathrm{C}$-APAP] derived from APAP was high in liver and kidneys. ${ }^{10)}$ Another study result shows that concentration values of APAP in various tissues including liver, the target organ of metabolism, were significantly high in the lung, liver, kidney. ${ }^{4}$ According to the our results, low uptake of radioactivity in the liver compared to previous studies was observed. The data related to the uptake of ${ }^{131} \mathrm{I}$ APAP in the lung and kidneys in rats agreed with these data.

APAP was observed to transfer from the gastrointestinal tract primarily by passive non-ionic diffusion and the rate of transfer depended on factors such as the concentration gradient between the gastrointestinal lumen and the circulation, aqueous and lipid soluble and mucosal blood flow, permeability and surface area. ${ }^{11,12)}$ We observed also that the highest uptake in the stomach $(p<0.05)$ was at $60 \mathrm{~min}$.

Recent studies which have shown that acetaminophen crosses blood-brain barrier support the view that this drug exerts its effect by a central mechanism. Our results indicate that ${ }^{131}$ I-APAP accumulate in some brain regions. The data indicated that the uptake of ${ }^{131}$ I-APAP was highest in the frontal cortex and mid-brain regions at $15 \mathrm{~min}$ (Fig. 2). On the other hand, the highest uptake of ${ }^{131}$ I-APAP in the cerebellum, hypothalamus, and medulla ponds were seen at $120 \mathrm{~min}$, and the highest uptake was observed in the hippocampus at $60 \mathrm{~min}$. In the study carried out by Courade et al., they used $\left[{ }^{3} \mathrm{H}\right]$-acetaminophen for a detailed analysis of the regional distribution of this drug in the CNS after i.v. administration in rats. Their study showed acetaminophen levels were high in cortexes (at $15-45 \mathrm{~min}$ ), striatum (at $45 \mathrm{~min}$ ), hippocampus (at $15 \mathrm{~min}$ ) and hypothalamus (at $45 \mathrm{~min}$ ). ${ }^{7)}$ The uptake of APAP in the medulla ponds and mid-brain was quite slowly clearance. ${ }^{13)}$ We found also similar results. In conclusion, the present data demonstrate the ability of acetaminophen to cross the blood-brain barriers as expected from the central action of this analgesic drug.

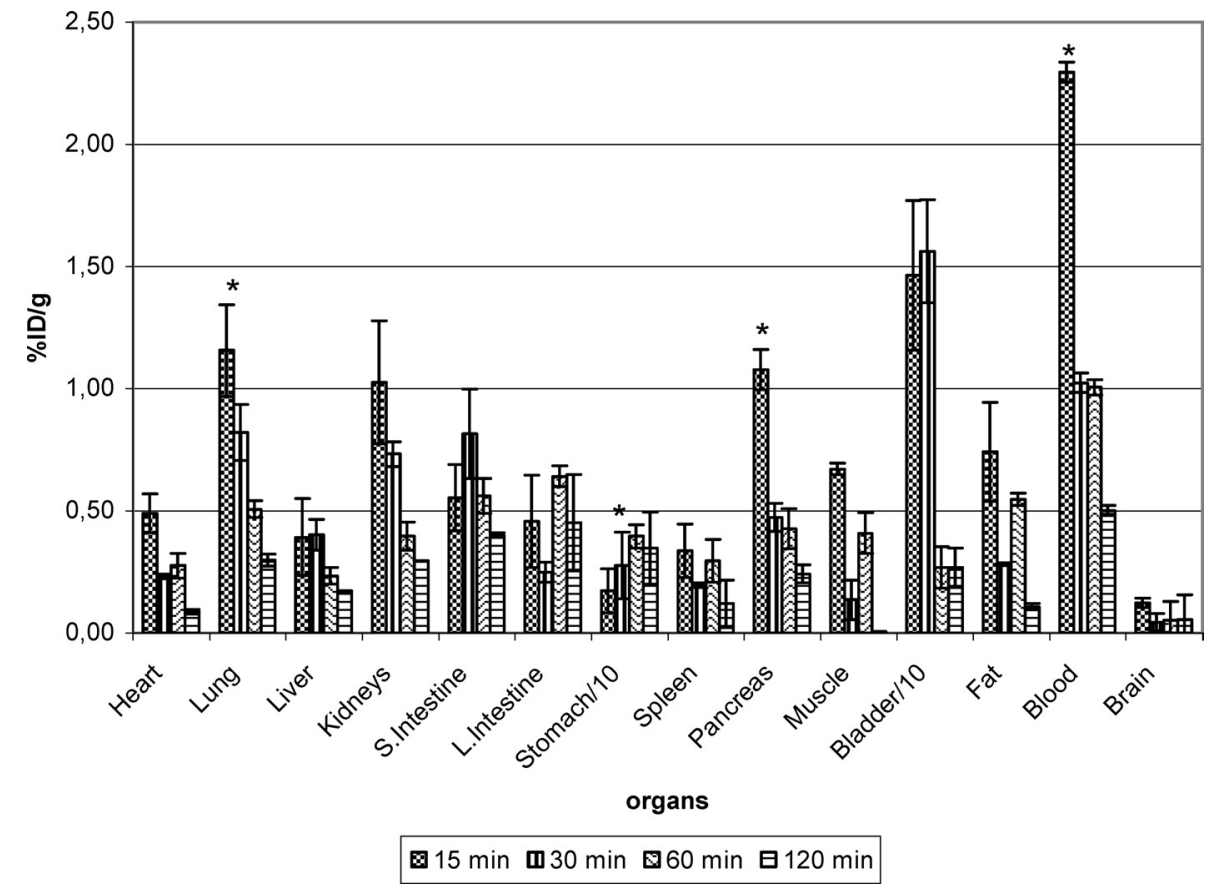

Fig. 1. Biodistribution of ${ }^{131} \mathrm{I}$-APAP in Rats as a Function of Time

$* p<0.05, \% \mathrm{ID} / \mathrm{g}$ : mean value \pm S.D. Stomach/10: $(\% \mathrm{ID} / \mathrm{g})$ value $/ 10$ for stomach. Bladder/10: $(\% \mathrm{ID} / \mathrm{g})$ value/10 for bladder. 


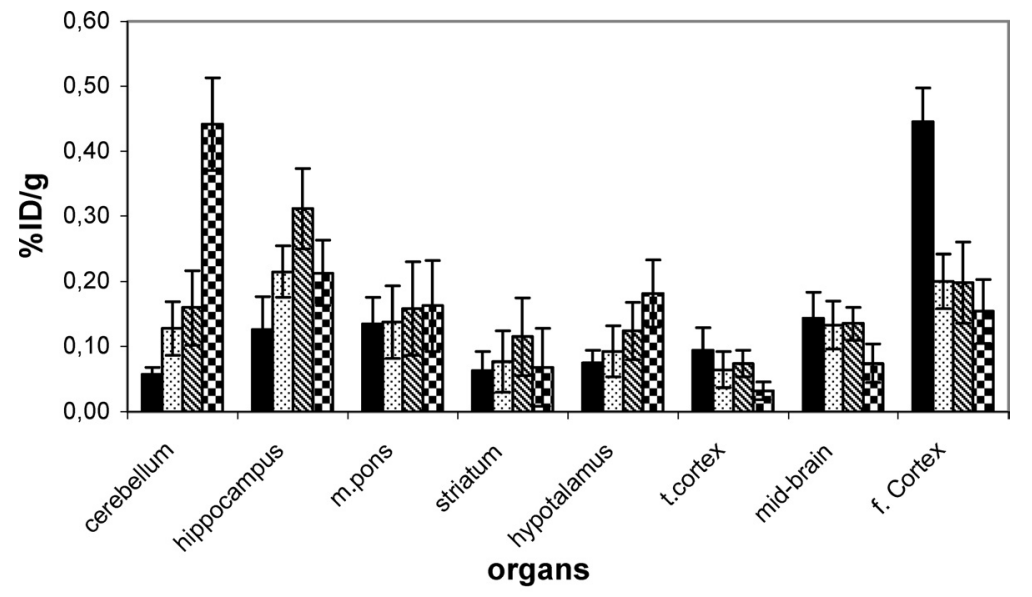

$15 \min 030 \min \$ 60 \min \mathbf{0} 120 \mathrm{~min}$

Fig. 2. Percent of Injected Activity of ${ }^{131}$ I-APAP per Gram Tissue

Short half-life isotopes such as C-11, F-18, N-13, O-15 and I-123 are used or radio synthesis is preferred for achieving high specific activity. In this study, the specific activity of ${ }^{131}$ I-APAP was $56.60 \mathrm{GBq} / \mathrm{mmol}$. Therefore APAP might be labeled with ${ }^{123} \mathrm{I}$ with a high specific activity. As a result, ${ }^{131} \mathrm{I}$ APAP might be a brain radiopharmaceutical and be used for SPECT images of the brain, in this case ${ }^{123} \mathrm{I}$ in place of ${ }^{131} \mathrm{I}$.

\section{Conclusions}

Experimental results indicated that the labeling yield was high $(94 \pm 4.0 \%)$. Furthermore ${ }^{131} \mathrm{I}$-APAP has enough stability for diagnostic scanning. As known, I-123 is an attractive radionuclide for SPECT imaging because it emits abundant $(85 \%) 159 \mathrm{keV}$ photons, which is suitable for gamma ray detectors (SPECT) and has a $13.3 \mathrm{~h}$ half-life which are ideal for imaging study. ${ }^{131}$ I-APAP showed that it can be uptaken in brain tissues and suggested that it might make a useful clinical candidate. In that case, labeling APAP with I-123 instead of I-131, may give a radiopharmaceutical which might be used for SPECT images of the brain.

\section{References}

1) Fairbrother J. E., Analytical Profiles of Drug Substances, 1, 3 (1974).

2) Sumioka I., Matsura T., Kai M., Yamada K., Life Sci., 74, 2551-2561 (2004).

3) Noriega G. O., Ossola J. O., Tomaro M. L., Batlle A. M. del C., Int. J. Biochem. Cell Biol., 32, 983-991 (2000).

4) Sawamoto T., Yuji K., Sasaki K., Kimura T., Nakayama T., Int. J. Pharm., 146, 181-191 (1997).

5) Wei L., Lancet, 361, 1560-1560 (2003).

6) Mugford C. A., Tarloff J. B., Drug Metab. Dispos., 23, 290-294 (1995).

7) Courad J. P., Besse D., Delchambre C., Hanoun N., Hamon M., Eschalier F., Caussade F., Cloarec A., Life Sci., 69, 1455-1464 (2001).

8) Alloui A., Chassaing C., Schmidt J., Ardid D., Dubray C., Cloarec A., Eschalier A., Eur. J. Pharmacol., 443, 71-77 (2002).

9) Yurt F., Unak P., Biber F. Z., J. Radional. Nucl. Chem., 251, 241-244 (2002).

10) Mugford C. A., Tarloff J. B., Toxicol. Lett., 93, 15-22 (1997).

11) Laurie P. F., "A Critical Bibliographic Review," 2nd ed., Vol. 4, Florence, KY, U.S.A., Routledge, 2001, pp. 45-50.

12) Laurie P. F., "A Critical Bibliographic Review," 2nd ed., Vol. 4, Florence, KY, U.S.A., Routledge, 2001, p. 87.

13) Sandrini M., Romualdi P., Capobianco A., Vitale G., Morelli G., Pini L. A., Candeletti S., Neuropeptides, 35, 110-116 (2001). 